\title{
Detection of Scene Change in Video
}

\author{
Chandra Shekhar Mithlesh ${ }^{1}$, Dolley Shukla ${ }^{2}$ \\ ${ }^{1}$ ME Scholar (Communication Engineering), Shri shankaracharya College of Engineering and Technology, Junwani, Bhilai, Dist-Durg, \\ Chhattisgarh 490020, India \\ ${ }^{2}$ Senior Associate Professor, Shri shankaracharya College of Engineering and Technology, Junwani, Bhilai, Dist-Durg, Chhattisgarh
} 490020, India

\begin{abstract}
A number of automated scene change detection methods for indexing a video sequence to facilitate browsing and retrieval have been proposed in recent years. Scene change detection plays an important role in a number of video applications, including video indexing, semantic features extraction, multimedia information systems, Video on demand (VOD), digital TV, online processing(networking) neural network mobile applications services and technologies, cryptography, and in watermarking. Recent advances in technology have made tremendous amount of multimedia content available. The amount of video is increasing, due to which the systems that improve the access to the video is needed. A current research topic on video includes video abstraction or summarization, video classification, video annotation and content based video retrieval. In all these applications one needs to identify shot and key frames in video which will correctly and briefly indicates the contents of video. This project emphasizes on video shot boundary detection using one of the methods of the color histogram wherein scaling of the histogram metrics is an added feature. The difference between the histograms of two consecutive frames is evaluated resulting in the metrics. Further scaling of the metrics is performed to avoid ambiguity and to enable the choice of apt threshold for any type of videos which involves minor error due to flashlight, camera motion, etc. Two sample videos are used here with pixels using color histogram approach in the video sequence. An attempt is made for the retrieval of color video. The purpose of this project is to develop a scene change detection system that can detect abrupt scene change in a video using one of the methods of the color histogram. The scene change detector is build by using MATLAB.
\end{abstract}

Keywords: Video, Video Sequence, Group of Picture(GOP), Frame, Histogram, wavelet statistics, Scene/Shot Boundary Detection(SCD), Video Retrieval, video indexing, browsing and retrieval. False Detection, Detection Rate, SCD, Recall \& Precision, PSNR, Macroblocks.

\section{Introduction}

Information databases have evolved from simple text to multimedia with video, audio, and text. The query mechanism for a video database is similar in concept compared to a textual database. Object searching is analogous to word searching; scene browsing is similar to paragraph searching; video indexing is comparable to text indexing or bookmarking. However, the implementation for video content based searching is very different and much more difficult than the query mechanism for a textual database. Scene change detection technique can often be applied for scene browsing and automatic and intelligent video indexing of video sequences for video databases. Once individual video scenes are identified, we can use content based indexing mechanisms (such as indexing by object texture, shape, color, motion) to index and query image contents in each video scene.

\subsection{General Video Structure}

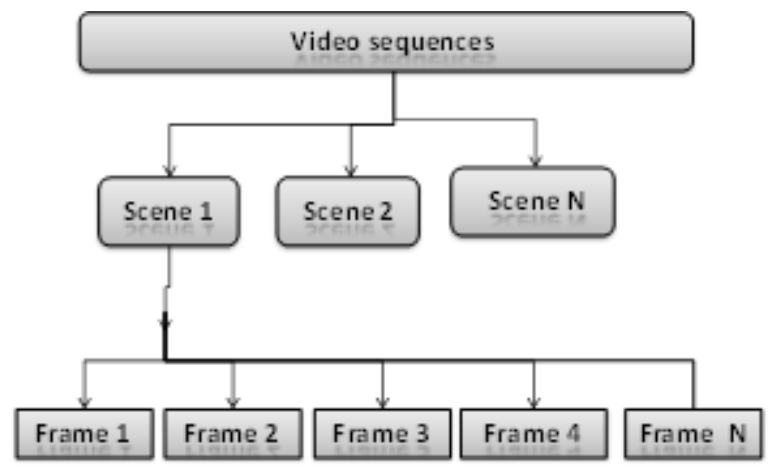

Figure 1.1: General Video Structure
Image: Images may be two-dimentional, such as photograph , screen display, and as well as a three-dimensional, such as hologram. They may be captured by optical such as cameras, mirror, lenses, telescope etc.

Picture Element: The pixel (a word invented from "picture element") is the basic unit of programmable color on a computer display or in a computer image

Video: A typical video sequence its organized into a sequence of group of pictures(GOP).A video can be broken down in scene, shot and frames. each GOP consists of one I (intra-coded) and a few $\mathbf{P}$ (predicted) and $\mathbf{B}$ (bi-directionally interpolated) frames as shown in Figure 1

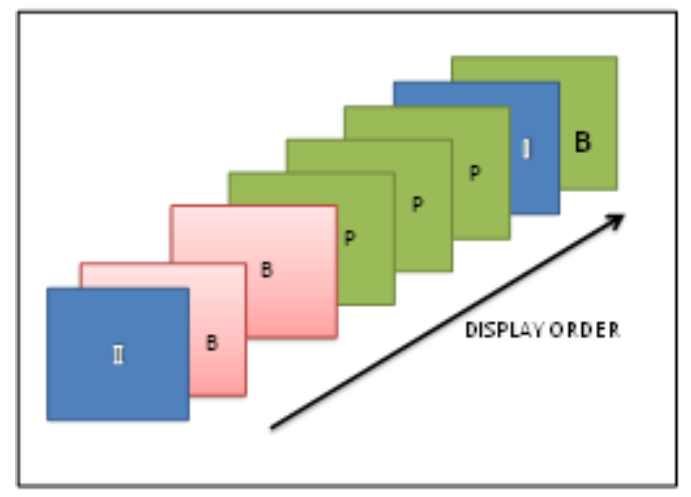

Figure 1.2: Group of Picture (GOP)

\section{I-Picture}

A I-picture is intra-frame coded and does not depend on any previous or future frames. The bit-rate of a I-picture depends on the visual content of the picture. If the picture is of high activity, then the bit-rate will be high. When a scene change occurs between two consecutive I-pictures, the content of the 


\section{International Journal of Science and Research (IJSR) \\ ISSN (Online): 2319-7064}

Index Copernicus Value (2013): 6.14 | Impact Factor (2014): 5.611

two I-pictures will be totally different. The transition may be from high-activity to low-activity, low-activity to highactivity, or with similar activity. Therefore, the bit-rate of the I-picture following the scene change will be increased, decreased or remained similarly. This implies that we cannot guarantee to detect all cut points if we only look for the significant change in total bit-rate of two consecutive Ipictures. However, when two pictures are of different content, they will have different local statistics even if their global characteristics are similar. Therefore, if we measure the bit-rate difference at macroblock level, we can extract the change of image content between two consecutive frames of sequence. A large change in bit-rate at macroblock level between two consecutive I-pictures means that there is a scene change between them.

\section{P-Picture}

P-picture is a forward motion-predicted frame, which depends on the previous P- or I-picture. When a scene change occurs between two consecutive P-pictures, it is difficult to make the forward prediction since the content of the current frame will

be totally different from the previous one. Most of the macroblocks have so large prediction error that they will be encoded using the intra-coded mode. This will of course lead to a significant increase in the bit-rate of the current frame. Therefore, we can detect the scene change by checking whether there is a large increment in the bit-rate of two consecutive P-frames.

\section{B-Picture}

B-picture is a bidirectional-predicted frame, which depends on both the previous and future P- or I-picture. When a scene change occurs, the content of the current frame will have large difference with the previous anchor frame but have similar content with the future one. Therefore, most of the macroblocks depend mostly on the future P- or I-picture and will be backward predicted. Since the prediction can only be done mostly in one direction, the coding efficiency of the motion compensation will be lower and it will lead to the increase in the bit-rate when compared with the previous Bpicture. However, such a increment will not be as significant as that occurred in I- or P-picture.

Scene: A scene is a logical grouping of shots into a semantic unit.

Shot: A shot is a sequence of frames captured by a single camera in a single continuous action. A shot boundary is the transition between two shots.

Frame: A digital video consists of frames that are a single frame consists of pixels.

\subsection{Video Segmentation Approach}

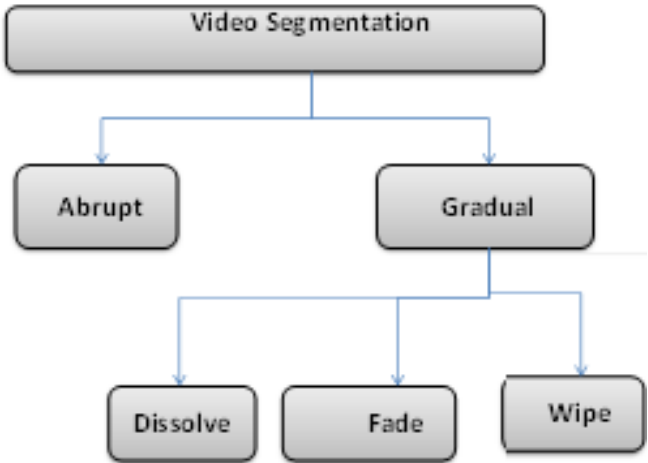

Figure 1.3: Video Segmentations Approach

\subsection{Video Segmentation}

There are mainly four different types of common shot boundaries within shots

1) Abrupt-Abrupt transition occurs in a single frame.

2) Gradual-It is again classified into 3 types.

Abrupt transitions are very easy to detect, as the two frames are completely uncorrelated. But, gradual transitions are more difficult to detect as the difference between frames corresponding to two successive shots is substantially reduced. They are

a) A Fade: Two different kinds of fades are used: The fadein and the fade-out. The fade-out emerges when the image fades to a black screen or a dot. The fade-in appears when the image is displayed from a black image. Both effects last a few frames.

b)A dissolve: It is a synchronous occurrence of a fade-in and a fade-out. The two effects are layered for a fixed period of time e.g. 0.5 seconds (12 frames). It is mainly used in live in-studio transmissions.

c) A wipe: This is a virtual line going across the screen clearing the old scene and displaying a new scene. It also occurs over more frames. It is commonly used in films such as Star Wars and TV shows. As these effects exist, shot boundary detection is a nontrivial task

\subsection{Shot Boundary}

The success of the segmentation approach depends largely on how well the video materials are divided into segments or shots. A shot is defined as a part of the video that results from one continuous recording by a single camera. A scene is composed of a number of shots, while a television broadcast consists of a collection of scenes. The gap between two shots is called a shot boundary.

\subsection{Scene Change Detection}

Analyzing the visual content of the video and partitioning it into a set of basic units called shots. This process is also referred to as video data segmentation. Content-based sampling thus can be approximated by selecting one representing frame from each shot, since a shot is defined as a continuous sequence of video frames which have no significant inter-frame difference in terms of their visual contents. A single shot usually results from a single continuous camera operation. This partitioning is usually achieved by sequentially measuring inter-frame differences 


\section{International Journal of Science and Research (IJSR) \\ ISSN (Online): 2319-7064 \\ Index Copernicus Value (2013): 6.14 | Impact Factor (2014): 5.611}

and studying their variances, e.g., detecting sharp peaks. This process is often called scene change detection (SCD).

\subsection{Boundaries Approaches}

There have been a number of various approaches to handle different scene/shot boundaries.

\section{Color Histograms}

The first approach tested at Dublin was a shot detection based on color histograms. They computed frame-to-frame similarities based on colors which appeared within them, albeit of the relative positions of those colors in the frame. After computing the inter-frame similarities, a threshold can be used to indicate shot boundaries. It needs dynamic threshold to work on other effects than simple shot boundaries.

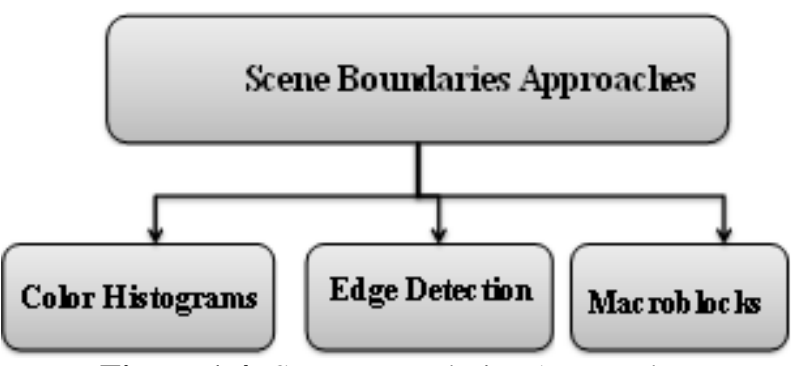

Figure 1.4: Scene Boundaries Approaches

\section{Edge Detection}

The next approach is Edge Detection which is based on detecting edges in two neighboring images and comparing these images. It should be possible to detect all kinds of shot boundaries by detecting the appearance of edges in a frame which are far away from the ones in the previous frame. The tested approach in Dublin used over 2 hours and 40 minutes of video files of different TV broadcasts. They spotted various reasons why their program missed a real cut between scenes:

- Blurred images where the edges could not be defined clearly

- Images with similar backgrounds or intensity edges to the next-following image

- Dark or bright images where the edges are not defined in an accurate manner

- Straight cuts from a blank screen to a dark screen

- A cut between different camera perspectives showing the same scene.

They also detected reasons for wrong identification of cuts:

- Fast action scenes with fast moving and changing edges

- Camera flashes

- Close-up moving scenes

- Objects moving in front of the camera lens without being present on the image before

- A zoom out or in, camera pan or any camera motion

- Computer generated scenes

- Interferences in the video from broadcasting or recording

- An object cut from an image

Main problems for missing cuts in all kinds of videos are cuts between dark scenes and the detection of so-called pseudo-cuts during the credits at the end of a film or programmed. They also found out that the detection of false shots increases with the quality and size of the example videos. Since many false detection had occurred because of camera panning and/or zooming they implemented a technique to compensate these movements. This solution can counter problems caused by dissolves and fades and other changes using soft color changes. The advantage - compared to colour based shot detection - is that this technique will not be fooled by colour changing effects like a flash. But on the other side, each frame has to be decoded, so it runs very slowly.

\section{Macroblocks}

Besides, they investigated the shot boundary detection using macroblocks. Depending on the types of the macroblock the MPEG pictures have different attributes corresponding to the macroblock. Macroblock types can be divided into forward prediction, backward prediction or no prediction at all. The classification of different blocks happens while encoding the video file based on the motion estimation and efficiency of the encoding. If a frame contains backward predicted blocks and suddenly does not have any, it could mean that the following frame has changed drastically which would point to a cut. This approach, however, becomes difficult to implement when there is a shot change, and the frame in the next shot contains similar blocks as the frame before.

Above are different techniques for video scene change detection and we are focusing on the method where in histogram of the color image we are scaling in order to get good result.

\section{Literature Review}

This chapter deals with the survey of various research papers that have contributed to solve the problem of scene change detection in video sequences. There is a growing demand of video indexing, scene browsing and retrievals in Signal Processing.

K. Tse, J. Wei et al. (1995) presented a scene change detection algorithm for mpeg compressed video sequences. Video is an important and challenge media and requires sophisticated indexing schemes for efficient retrieval from visual databases. An important step in video indexing is scene change detection. Recently, several scene change detection algorithms in the pixel and compressed (MPEG-2) domains have been reported in the literature. These algorithms are computationally complex and are not very robust in detecting gradual scene changes In this paper, an efficient technique for detecting scene changes in the MPEG-2 compressed domain. The proposed algorithm has the advantage of fast scene change detection. In addition, this algorithm has the potential to detect gradual scene changes. This algorithm is three times faster than its pixel domain counterpart. In addition, it only requires $10 \%$ of storage compared to the IDMn algorithm.[1]

Jian Feng et al. (1996) developed a new scene change detection algorithm for MPEG encoded video sequences. The proposed method is very simple that it only requires the bit-rate information at macro block level as well as the number of various motion-predicted blocks for detection. 


\section{International Journal of Science and Research (IJSR) \\ ISSN (Online): 2319-7064 \\ Index Copernicus Value (2013): 6.14 | Impact Factor (2014): 5.611}

Those information can be extracted easily from the encoded bit-stream without decompression. The proposed algorithm is effective for detecting abrupt scene changes in MPEG video. [2]

\section{Limitations}

- Need to record the number of bits required by each macro block as well as the number of different motion-predicted blocks.

- The proposed algorithm is effective in detecting abrupt scene changes in MPEG video sequences but not perfect.

Ralph M. Ford et al.(1997) presented a five metrics for scene change detection in video sequences. Metric previously applied to this problem are surveyed, and are quantitatively compared to the new metrics. The proposed metrics are superior. These metrics were also applied to the detection of gradual transitions. $\mathrm{y}$ is a good global metric for detecting abrupt cuts. [3]

\section{Limitations}

The F-test and vi-3 perform the best overall for abrupt cuts, but require more computation time.

Global metric did not perform well for gradual transitions, but the statistic based metrics did.

Taehwan Shin et al.(1998) presented a hierarchical scene change detection in an mpeg-2 compressed video sequence. In this paper, we propose an efficient scene change detection algorithm for direct processing of MPEG-2 video bitstreams. The proposed algorithm utilizes the hierarchical structure of the compressed bitstreams and statistical characteristics of the coded parameters, thus greatly reducing computational requirement compared to pixel domain processing with full decompression. Occurrence of scene change is checked first in a COP level, and if the result is affirmative it is checked again in lower levels : sub-GOP and each picture. We used several metrics for different levels : variance of DC images for I-pictures, number of macroblock types for P-pictures and motion vector types for $\mathrm{B}$ pictures. The proposed algorithm uses such features of the video which are easily extracted by minimal decoding of the input bitstream that very efficient processing is achieved. [4]

\section{Limitations}

- In this algorithm, scene change is checked in a hierarchical fashion from GOP to sub-GOP and picture level, thus reducing much of the processing requirements.

W.A.C. Fernando et al. (1999) presented a Wipe Scene Change Detection in Video Sequences. This paper presents a novel algorithm for wipe scene change detection in video sequences. In the proposed scheme, each image in the sequence is mapped to a reduced image. Then we use statistical features and structural properties of the images to identify wipe transition region. Finally, Hough transform is used to analyze the wiping pattern and the direction of wiping .The algorithm is capable of detecting all wipe regions accurately even when the video sequence contains other special effects like fading, dissolving, panning the proposed algorithm can be used in uncompressed video to detect wipe regions with a very high reliability. [5]

\section{Limitations}

- Further work is required to extend this algorithm for compressed video.

W.A.C. Fernando, et al. (2000) presented a unified approach to scene change detection in uncompressed. In this paper, a novel algorithm for scene change detection in video sequences. There is an urgent need to extract key information automatically from video for the purposes of indexing, fast retrieval and scene analysis. To support this vision, reliable scene change detection algorithms must be developed. Results on video of various content types are reported and validated with the proposed scheme in uncompressed and MPEG-2 compressed video. the accuracy of the detected transitions is above 95\% and 90\% for uncompressed and MPEG-2 compressed video respectively. The proposed algorithm can be used in uncompressed and compressed video to detect scene changes with a high reliability. The algorithm is capable of detecting all scene changes accurately. [6]

\section{Limitations}

- Future work is required to extend the algorithm for camera movements detection within the same framework.

Young-Min Kim et al. (2000) presented a fast scene change detection using direct feature extraction from mpeg compressed videos. In order to process video data efficiently, a video segmentation technique through scene change detection must be employed. Many of advanced video applications require manipulations of compressed video signals. So, the scene change detection process is achieved by analyzing the video directly in the compressed domain, thereby avoiding the overhead of decompressing video into individual frames in the pixel domain. In this paper, we propose a fast scene change detection algorithm using direct feature extraction from MPEG compressed videos, and evaluate this technique using sample video data. This process was made possible by a new mathematical formulation for deriving the edge information directly from the DCT coefficients. The proposed algorithm is comparable to the DC method in speed, and was found to be five to six times faster than the FB method.[7]

\section{Limitations}

- More mathematical calculation is needed.

Wensheng Zhou,et al.(2000) presented an On-line Scene Change Detection of Multicast Video. Network-based computing is becoming an increasingly important area of research, whereby computational elements within a distributed infrastructure process/enhance the data that traverses through its path. These computations as online processing and this paper investigates scene change detection in the context of MBone based proxies in the network. Online processing varies from traditional offline processing schemes, where for example, the whole video scope is known as a priori, which allows multiple scans of the stored video files during video processing. The proposed algorithms do scene change detection and extract key frames from video bitstreams sent through the mbone network. Several algorithms based on histogram differences and evaluate them 


\section{International Journal of Science and Research (IJSR) \\ ISSN (Online): 2319-7064 \\ Index Copernicus Value (2013): 6.14 | Impact Factor (2014): 5.611}

with respect to precision, recall, and processing latency a few effective methods of on-line video scene change detection over MBone video for the purposes of annotation/filtering. Main advantages of our algorithms is their ability to support real-time video processing on the network. Joint algorithms based on video codec characteristics for acquiring fast and accurate scene detection were carried out. Both global color histograms were extracted and block information of DCT coding was used.. Algorithms are capable of satisfying online annotation needs. These algorithms on different data bandwidths, we are able to choose the right algorithm with the best performance in each case.[8]

\section{Limitations}

- Algorithms are mainly targeting real-time video scene analysis, improving processing speed and reducing latency are two more issues of concern, which makes it improper to compare our algorithms with other off-line scene detection techniques, such as those we mentioned in the Related Work section, where video data are processed offline.

- In the future, they plan to study more video on-line annotations based on video content, such as key frame classification. Because of the complicated nature of video processing, they plan on using multiple workstations working in parallel to realize more sophisticated real-time annotation

- Plan to study algorithms that are tolerant of packet losses in the network.

- The on-line processing system is designed to meet the requirements of real-time video multicasting over the Internet and to utilize the successful video parsing techniques available today.

Chung-Lin Huang, et al.(2001) presented a robust scenechange detection method for video segmentation. This paper proposes a new method that combines the intensity and motion information to detect scene changes such as abrupt scene changes and gradual scene changes. Two major features are chosen as the basic dissimilarity measures, and self and cross-validation mechanisms are employed via a static scene test. Developed a novel intensity statistics model for detecting gradual scene changes. the proposed algorithms are effective and outperform the previous approaches. The advantages of our method over the conventional threshold problem in avoiding the false alarms by using the validation mechanism. It also proves that the statistical model-based approach is reliable for gradual scene-change detection. Experimental results show that a very high detection rate is achieved. [9]

\section{Limitations}

- The false alarm rate is comparatively low but improvement is required.

Shu-Ching Chen,et al.(2002 ) presented a change detection by audio and video clues. Automatic video scene change detection is a challenging task. Using audio or visual information alone often cannot provide a satisfactory solution. However, how to combine audio and visual information efficiently still remains a difficult issue since there are various cases in their relationship due to the versatility of videos. In this paper, they presented an effective scene change detection method that adopts the joint evaluation of the audio and visual features. First, video information is used to find the shot boundaries. Second, the audio features for each video shot can be extracted. Lastly, an audio-video combination schema is proposed to detect the video scene boundaries. Unlike the traditional methods that first analyze audio and video data separately and then combine them, we analyze them at different phases The audio feature extraction is based on the detected video shots, which tends to be more stable and more reliable in charactering the audio data. The experimental results demonstrate that our method performs very well in terms of precision and recall value.[10]

Dan Lelescu et al (2003) presented a statistical sequential analysis for real-time video scene change detection on compressed multimedia bitstream. These algorithms must offer capabilities related to browsing, indexing, and retrieval of relevant data. A crucial step in multimedia processing is that of reliable video segmentation into visually coherent video shots through scene change detection. Video segmentation enables subsequent processing operations on video shots, such as video indexing, semantic representation, or tracking of selected video information. Since video sequences generally contain both abrupt and gradual scene changes, video segmentation algorithms must be able to detect a large variety of changes. While existing algorithms perform relatively well for detecting abrupt transitions (video cuts), reliable detection of gradual changes is much more difficult. In this paper, a novel one-pass, real-time approach to video scene change detection based on statistical sequential analysis and operating on compressed multimedia bitstream is proposed. video sequences as stochastic processes, with scene changes being reflected by changes in the characteristics (parameters) of the process. Statistical sequential analysis is used to provide an unified framework for the detection of both abrupt and gradual scene changes..[11]

\section{Limitations}

- For increased efficiency, the dimensionality of the original video data is reduced using an optimal transformation, that retains the most representative features of the original data, resulting in a new low-dimensional representation.

Xiaoquan Yi et al.(2005) presented a Fast Pixel-Based Video Scene Change Detection. This paper proposes a new simple and efficient method to detect abrupt scene change based on only pixel values. Conventional pixel-based techniques can produce a significant number of false detections and missed detections when high motion and brightness variations are present in the video. To increase scene change detection accuracy yet maintaining a low computational complexity, a two-phase strategy is developed. Frames are firstly tested against the mean absolute frame differences (MAFD) via a relaxed threshold, which rejects about $90 \%$ of the non-scene change frames. The rest $10 \%$ of the frames are then normalized via a histogram equalization process. A topdown approach is applied to refine the decision via four features: MAFD and three other features based on normalized pixel values - signed difference of mean absolute frame difference (SDMAFD*), absolute difference of frame variance $\left(\mathrm{ADFV}^{*}\right)$, and mean absolute frame differences 


\section{International Journal of Science and Research (IJSR) \\ ISSN (Online): 2319-7064 \\ Index Copernicus Value (2013): 6.14 | Impact Factor (2014): 5.611}

(MAFD*) method contributes to higher detection rate and lower missed detection rate while maintaining a low computational complexity, which is attractive for real-time video applications. Method is relatively immune from sharp illumination changes, moving objects, camera motion, and other similar effects because of the well combined metrics. Furthermore, our method uses only frame pixel values without any motion estimation processes so that frugal computational complexity is maintained, which makes it very attractive for real-time video applications. [12]

\section{Limitations}

- This technique is simple but it performs poorly in cases of high motions of objects, high motions of cameras, or severe scene luminance variations (i.e., flicker)

Gao, J. Jiang et al.(2006) presented a PCA-based approach for video scene change detection on compressed video. An automatic, real-time detection approach to video scene change detection is presented. Owing to the high correlation of two consecutive video frames, it is proposed that only the eigenvector corresponding to the largest eigenvalue is retained in the principal component analysis (PCA) for video data. A one-dimensional PCA feature of video data is then generated from the PCA. It shows superior performance compared to the histogram feature and the pixel feature.The detection algorithm based on this PCA feature is then designed to detect both abrupt and gradual transitions. The proposed approach is tested on the TREC video test repository to validate its performance. The superior performance of the new PCA feature. Then our change detection algorithm is based on the PCA feature to detect the scene change. [13]

\section{Limitations}

- Tested on the TREC video test repository to validation for its performance.

J.-R. Ding et al. (2007) presented adaptive group-of-pictures and scene change detection methods based on existing h.264 advanced video coding information. The H.264 advanced video coding (H.264/AVC) standard provides several advanced features such as improved coding efficiency and error robustness for video storage and transmission. In order to improve the coding performance of H.264/AVC, coding control parameters such as group-of-pictures (GOP) sizes should be adaptively adjusted according to different video content variations (VCVs), which can be extracted from temporal deviation between two consecutive frames. The authors present a simple VCV estimation to design adaptive GOP detection (AGD) and scene change detection (SCD) methods by using the obtained motion information, where the motion vectors and the sum of absolute transformed differences as VCV features are effectively used to design the AGD and SCD algorithms, respectively. In order to avoid unnecessary computation, the above VCV features are obtained only in the $4 \times 4$ inter-frame prediction mode. the proposed AGD with SCD methods can increase the peak signal-to-noise ratio by $0.62 \mathrm{~dB}$ on average over the H.264/AVC operated with a fixed GOP size. Besides, the proposed SCD method can reach a scene change detection rate of $98 \%$.On the other hand, if the scene-changed frame without any detection mechanism is coded by intercoding. It will become inefficient and waste considerable computation time in motion estimation. We found that the GOP size for H.264/AVC should be appropriately decreased and increased for higher and lower VCV frames, respectively, to improve the coding performance. In this paper, we successfully utilized the existing video coding information, such as motion vectors and motion residuals, to become an effective VCV feature. Both the proposed AGD and SCD methods can effectively help to adjust a better GOP size to improve the coding performance of H.264/AVC. the proposed joint AGD and SCD schemes can increase PSNR by at least $0.62 \mathrm{~dB}$ compared with the H.264/AVC operated in the fixed GOP size on average. [14]

\section{Limitations}

- The proposed and some existing VCV characteristics can be further extended for adaptive search window, adaptive search methods (of three step search and

ZHANG Ji et al.(2007) presented an effective error concealment framework for h.264 decoder based on video scene change detection. In this paper, we propose an effective error concealment framework for H.264 decoder based on the scene change detection. The proposed framework quickly and accurately detects whether scene change occurs in the decoding frame, based on the detection result, both corrupted intra frames and damaged inter frames can be reconstructed by spatial or improved temporal EC (Error Concealment) algorithm. The experiment shows that, compared with the traditional error concealment method in the H.264/A VC non normative decoder, the proposed framework has better robustness and can efficiently improve the visual quality and PSNR of the decoded video. [15]

\section{Limitations}

- The final experimental results demonstrate that our framework can apparently improve the visual quality of reconstructed video sequence and PSNR.

- In the future, they will emphasize more on exploring the scene change algorithm for B frames and improve spatial or temporal EC algorithms in the new frame work.

Jens Brandt et al.(2008) presented a fast frame-based scene change detection in the compressed domain for mpeg-4 video. Detection of scene changes is an elementary step in automatic video processing like indexing, segmentation or transcoding Video indexing and segmentation allow fast browsing without decoding the complete video. In the case of transcending, the information about scene changes such as cuts and fades as well as about special movements like rotations or zooms in video frames is helpful to determine suitable transcoding parameters. In the compressed domain only information about DCT values as well as motion information can be used to determine such scene changes and movements. Therefore we defined different measures which use the encoded DCT values and motion vectors of each compressed frame. Based on these measures as well as on motion vector histograms, present a fast approach to detect different kinds of scene changes and special movements in MPEG-4 videos in the compressed domain In this paper we have presented a fast frame-based algorithm for compressed domain scene change detection in MPEG-4 video streams. The easy computation of the used metrics and 


\section{International Journal of Science and Research (IJSR) \\ ISSN (Online): 2319-7064 \\ Index Copernicus Value (2013): 6.14 | Impact Factor (2014): 5.611}

histograms makes the whole scene change detection algorithm very fast which is very important for real time video processing. Therefore the scene change detection can only be a tool to indicate a stronger or weaker correlation towards one or the other scene movement. However, the evaluation results show that the algorithm detects a high number of scene changes and movements. Based on these promising results we are currently developing a method to use the information about detected scene changes and special movements within a video stream for determining suitable transcoding parameters. [16]

\section{Limitations}

- The highly statistical nature of the scene change detection results in a statement that by design cannot be absolutely correct.

Hakan Haberdar et al.(2010) presented a disparity map refinement for video based scene change detection using a mobile stereo camera platform. This paper presents a novel disparity map refinement method and vision based surveillance framework for the task of detecting objects of interest in dynamic outdoor environments from two stereo video sequences taken at different times and from different viewing angles by a mobile camera platform. Preliminary disparity images are refined based on estimated disparities in neighboring frames. Segmentation is performed to estimate ground planes, which in turn are used for establishing spatial registration between the two video sequences. Finally, the regions of change are detected using the combination of texture and intensity gradient features. We present experiments on detection of objects of different sizes and textures in real videos. The framework is evaluated on 4 real video sequences and the experimental results show that the proposed framework is able to detect changes/objects of different sizes and textures, and is able to ascertain if there are no changes in the scene, thereby minimizing false positives. [17]

\section{Limitations}

- This method need for future work and it will focus on relaxing our assumption on known temporal alignment and improving the spatial registration module.

Ufuk Sakarya et al.(2010) presented a video scene detection using graph-based representations. One way of realizing this step is to obtain shot or scene information. One or more consecutive semantically correlated shots sharing the same content construct video scenes. On the other hand, video scenes are different from the shots in the sense of their boundary definitions; video scenes have semantic boundaries and shots are defined with physical boundaries. In this paper, we concentrate on developing a fast, as well as wellperformed video scene detection method. Our graph partition based video scene boundary detection approach, in which multiple features extracted from the video, determines the video scene boundaries through an unsupervised clustering procedure. For each video shot to shot comparison feature, a one-dimensional signal is constructed by graph partitions obtained from the similarity matrix in a temporal interval. After each one-dimensional signal is filtered, an unsupervised clustering is conducted for finding video scene boundaries.We adopt two different graph-based approaches in a single framework in order to find video scene boundaries. The proposed graph-based video scene boundary detection method is evaluated and compared with the graphbased video scene detection method presented in literature. [18]

\section{Limitations}

- Some issues are considered into account for future research directions. Different video features can be used in order to improve the results and; moreover, content varying based changes in the multiple features may increase the scene detection performance.

- Especially, feature weighting in clustering process can also be applied. In addition, an adaptation of the proposed method for other types of videos, like news, documentaries, etc., is another research direction.

Chung-chi Lin et al.(2013) presented A Video De-interlacing with Precise Inter field Information by Hybrid Scene Change Detection.This paper presents a motion adaptive deinterlacing technique with precise interfield information by global and local scene changes detection. Scene changes happen fairly often in film broadcasting and they tend to destabilize the quality of performance while de-interlacing technique is utilized. The proposed method utilizes the low computational complexity of de-interlacing technique to improve video sequences on the progressive devices. Our hybrid scene change detection method for de-interlacing uses the precise interfield information as interpolation. The simulation results indicate that increasing the precision and stability of the interfield information promotes quality of video sequence. the proposed method presents a higher quality of video sequences than other interpolation methods.[19]

Ankita P. Chauhan1 et al (2013) presented Hybrid Approach for Video Compression Based on Scene Change Detection. In order to fulfill the requirement of limited channel bandwidth and of growing video demand like streaming media delivery on internet, and digital library, video compression is necessary. In video compression, temporal redundancy between adjacent frames is removed with block based motion estimation algorithms. Video represents a sequence of frames captured from camera. Scene is a series of consecutive frames captured from narrative point of view. In this paper we present an effective scene change detection method for an uncompressed video. We have divided frames in to blocks and applied a canny edge detector in consecutive frames. Count no of pixels (ones) in each block and compare it with consecutive frames. If scene change happens then number of pixels per block will change, based on that change we can detect scene change in consecutive frame. presented a hybrid approach, in which we have used scene change detection along with block based motion estimation algorithms (BME) to compress video.In this paper we have presented a simple approach with high accuracy. To reduce the number of computations, hybrid approach along with scene change detection have been implemented.[20]

\section{Limitations}

- Simulation result shown that Proposed Hybrid approach compared with NTSS, 4SS and DS algorithm, it reduces 


\section{International Journal of Science and Research (IJSR) \\ ISSN (Online): 2319-7064 \\ Index Copernicus Value (2013): 6.14 | Impact Factor (2014): 5.611}

the cost of computational complexity and also gives approximately same PSNR result.

Soongi Hong et al (2013) presented Adaptive Thresholding for Scene Change Detection. Increasing the demands of digital video by developing the Internet market and the multimedia technologies, video indexing technique such as scene change detection is required to manage the data efficiently. In conventional methods, scene change is detected by comparing the value of current detected measure with the fixed threshold induced from preceding experiments. To solve this problem, a novel adaptive threshold decision method is proposed. First, histogram of scene change detection measure induced from preceding experiments is derived. Then this is modeled with lognormal distributed PDF and the model parameters are estimated. Consequently, experimental results obtained from this PDF model and estimated parameters demonstrate better performance of the proposed method comparing with conventional method. In this paper, we propose a novel adaptive threshold decision method based on statistical model and analysis. Using the estimated model function and model parameters from preceding simulations, and the calculated mean and variance of scene change detection measure from each video sequences, the threshold is dynamically allotted to each video sequences. Thus, our proposed method is stable in scene change detection ability on various video sequences[21]

Neetirajsinh J. Chhasatia et al. (2013) presented a Performance Evaluation of Localized Person Based Scene Detection and Retrieval in Video. In this paper work regarding person localization using novel approach has been carried out. Person localization is the first step in all the surveillance application in multimedia and human-computer interface applications. The objective of this paper is to address a system for a person localization system which describes important tasks of video content analysis, namely, video segmentation, localizing the person and their identification. A novel framework is proposed for combined video segmentation and retrieval of scene with object/person localization. In which the dominant regions as a large area of 2.2 Comparatively Study of Different SCD Algorithms the frame have been tracked throughout a shot and then stable features are extracted. Dominant region based effective parameters (DREP) model which includes low level features of the proposed model. Here the low level features are referenced as the color of dominant region, edges, area, centroid and position of a particular object or person to be localized in the video. Based on the extracted information dimensional processing is used to extract images of faces or the required objects. The simulated results based on the presented algorithm shows good performance. Also it is highly robust to camera, objects motions and can withstand illumination changes even in different poses in the sequences of the images.[22]

\section{Limitations}

- Improvement in its basic inputs would result in better performance of all these techniques.

S. Argyropoulos et al. (2013) presented a scene change detection in encrypted video bit streams. a novel method to detect scene changes in encrypted video streams is presented. Typically, in IPTV systems, the media stream is transmitted in encrypted form, and therefore the only available information to determine the scene changes are the packet headers which transport the video signal. Thus, the proposed method estimates the size and the type of each picture of the video sequence by extracting information from the packet headers. Then, based on the GOP structure, a set of rules are determined to predict changes of frame sizes which are indicative of scene changes in the video sequences. Furthermore, the application of the proposed method in the recently standardized ITU-T Recommendation P.1201.2 for no-reference audio-visual quality assessment for IPTV-grade services is presented to highlight how such method could be deployed. Finally, the proposed method is evaluated on a large set of video databases to demonstrate the validity of the proposed method.[23]

\section{Limitations}

- The proposed method can achieve a recall rate of $70 \%$.

Table 1: Comparatively study of different SCD algorithms

\begin{tabular}{|c|c|c|c|c|c|c|}
\hline \multirow[t]{2}{*}{ S.N } & \multirow[t]{2}{*}{ Name Of Journal } & \multirow[t]{2}{*}{ Year } & \multirow[t]{2}{*}{ Area } & \multirow{2}{*}{$\begin{array}{c}\text { Methodology } \\
\text { Used }\end{array}$} & \multicolumn{2}{|c|}{ Description } \\
\hline & & & & & Advantages & Limitation \\
\hline 1 & $\begin{array}{l}\text { A Scene Change } \\
\text { Detection } \\
\text { Algorithm for } \\
\text { MPEG } \\
\text { Compressed } \\
\text { Video Sequences }\end{array}$ & 1995 & $\begin{array}{c}\text { Multimedia } \\
\text { Information } \\
\text { Systems }\end{array}$ & $\begin{array}{l}\text { a novel algorithm for } \\
\text { scene } \\
\text { change detection using } \\
\text { the motion vectors and } \\
\text { DCT } \\
\text { coefficients in the } \\
\text { MPEG compressed } \\
\text { domain }\end{array}$ & $\begin{array}{c}\text { algorithm has the potential to } \\
\text { detect gradual scene changes } \\
\text { three times faster than its pixel } \\
\text { domain counterpart. } \\
\text { it only requires } 10 \% \text { of storage } \\
\text { compared to the IDMn } \\
\text { algorithun } \\
\text { fast scene change detection. }\end{array}$ & \\
\hline 2 & $\begin{array}{l}\text { Scene Change } \\
\text { Detection } \\
\text { Algorithm for } \\
\text { MPEG Video } \\
\text { Sequence }\end{array}$ & 1996 & $\begin{array}{c}\text { Video on } \\
\text { demand (VOD) } \\
\text { Digital TV }\end{array}$ & $\begin{array}{c}\text { a scene change } \\
\text { detection algorithm }\end{array}$ & $\begin{array}{c}\text { method is very simple } \\
\text { only requires the bit-rate } \\
\text { information } \\
\text { at macro block level } \\
\text { the number of various motion- } \\
\text { predicted blocks }\end{array}$ & $\begin{array}{l}\text { Need to record the } \\
\text { number of bits required by } \\
\text { each macro block as well } \\
\text { as the number of } \\
\text { different motion-predicted } \\
\text { blocks. } \\
\text { algorithm is effective in } \\
\text { detecting abrupt scene }\end{array}$ \\
\hline
\end{tabular}




\section{International Journal of Science and Research (IJSR) \\ ISSN (Online): 2319-7064}

Index Copernicus Value (2013): 6.14 | Impact Factor (2014): 5.611

\begin{tabular}{|c|c|c|c|c|c|c|}
\hline & & & & & $\begin{array}{l}\text { the proposed algorithm is } \\
\text { effective }\end{array}$ & $\begin{array}{l}\text { changes in MPEG video } \\
\text { sequences but not perfect }\end{array}$ \\
\hline 3 & \begin{tabular}{|c|} 
Metrics for Scene \\
Change Detection \\
in Digital Video \\
Sequences
\end{tabular} & \begin{tabular}{|l|}
1997 \\
\end{tabular} & $\begin{array}{l}\text { automatic } \\
\text { indexing of } \\
\text { video data }\end{array}$ & $\begin{array}{l}\text { a new content-based } \\
\text { scene } \\
\text { change detection } \\
\text { method }\end{array}$ & $\begin{array}{l}\text { metrics are superior } \\
\text { detection of gradual } \\
\text { transitions. } \\
\text { good global metric for } \\
\text { detecting abrupt cuts. }\end{array}$ & $\begin{array}{l}\text { perform the best overall } \\
\text { for abrupt cuts, but } \\
\text { require more computation } \\
\text { time. } \\
\text { did not perform well for } \\
\text { gradual transitions, but the } \\
\text { statistic based metrics did. }\end{array}$ \\
\hline 4 & \begin{tabular}{c|} 
Hierarchical \\
Scene Change \\
Detection In An \\
Mpeg-2 \\
Compressed \\
Video Sequence \\
\end{tabular} & \begin{tabular}{|l|}
1998 \\
\end{tabular} & \begin{tabular}{|c|} 
automatic Signal \\
Processing - \\
video \\
indexing, scene \\
browsing and \\
retrievals \\
\end{tabular} & \begin{tabular}{|} 
scene change detection \\
through the three \\
decision stages: GOP \\
algorithm A, sub-GOP \\
algorithm and picture
\end{tabular} & $\begin{array}{l}\text { very efficient processing is } \\
\text { achieved. }\end{array}$ & \begin{tabular}{|c|} 
scene change is checked \\
in a hierarchical fashion \\
from GOP to sub-GOP \\
and picture level, thus \\
reducing much of the \\
processing requirements.
\end{tabular} \\
\hline 5 & \begin{tabular}{|c|} 
Wipe Scene \\
Change Detection \\
in Video \\
Sequences
\end{tabular} & 1999 & \begin{tabular}{|c|} 
Signal \\
Processing - \\
communication \\
and \\
entertainment \\
\end{tabular} & \begin{tabular}{|c|} 
statistical feature based \\
approach for \\
wipe detection
\end{tabular} & \begin{tabular}{|c|} 
Accurately \\
very high reliability \\
scheme is very sensitive to the \\
type of the video sequence
\end{tabular} & $\begin{array}{l}\text { Further work is required } \\
\text { to extend this algorithm } \\
\text { for compressed video }\end{array}$ \\
\hline 6 & \begin{tabular}{|c|} 
A Unified \\
Approach To \\
Scene Change \\
Detection In \\
Uncompressed \\
And Compressed \\
Video"
\end{tabular} & 2000 & $\begin{array}{c}\text { Signal } \\
\text { Processing }\end{array}$ & $\begin{array}{l}\text { a novel unified } \\
\text { algorithm }\end{array}$ & \begin{tabular}{|} 
An efficient algorithm \\
detecting abrupt transitions \\
and gradual transitions \\
the accuracy of the detected \\
transition region \\
is above 98\% and above 95\% \\
for MPEG-2 compressed \\
video \\
scheme is less complex. \\
high reliability
\end{tabular} & \\
\hline 7 & \begin{tabular}{|c|} 
Fast Scene \\
Change Detection \\
using Direct \\
FeatureExtraction \\
from MPEG \\
Compressed \\
Videos
\end{tabular} & 2000 & \begin{tabular}{|c|} 
Signal \\
Processing- \\
video on demand \\
(VOD),
\end{tabular} & $\begin{array}{l}\text { a fast scene change } \\
\text { detection } \\
\text { algorithm }\end{array}$ & $\begin{array}{c}\text { five to six times faster than the } \\
\text { FB (feature-based algorithm) } \\
\text { method }\end{array}$ & $\begin{array}{c}\text { Need for more } \\
\text { mathematical calculation }\end{array}$ \\
\hline 8 & $\begin{array}{l}\text { Scene change } \\
\text { detection } \\
\text { algorithms for } \\
\text { content based } \\
\text { video indexing } \\
\text { and retrieval } \\
\end{array}$ & $\begin{array}{l}\text { JUNE } \\
2001\end{array}$ & \begin{tabular}{|c|} 
Signal \\
Processing \\
(video indexing \\
and retrieval)
\end{tabular} & $\begin{array}{l}\text { a real-time algorithm/ } \\
\text { a novel algorithm } \\
\text { /sudden scene change } \\
\text { detection }\end{array}$ & $\begin{array}{l}\text {-fade and dissolve scene } \\
\text { change detection in video } \\
\text { sequences } \\
\text { - effects can be identified } \\
\text { accurately } \\
\end{array}$ & $\begin{array}{c}\text { More work is required on } \\
\text { the identification of large } \\
\text { local and global motion in } \\
\text { the video sequences }\end{array}$ \\
\hline 9 & \begin{tabular}{|c|} 
A Robust Scene- \\
Change Detection \\
Method for Video \\
Segmentation
\end{tabular} & \begin{tabular}{|l|} 
DEC \\
2001
\end{tabular} & $\begin{array}{c}\text { Signal } \\
\text { Processing } \\
\text { (Video } \\
\text { Segmentaio) }\end{array}$ & $\begin{array}{l}\text { Robust Scene-Change } \\
\text { Detection Method }\end{array}$ & $\begin{array}{l}\text {-a very high detection rate. } \\
\text {-the statistical model-based } \\
\text { approach is reliable for } \\
\text { gradual scene-change } \\
\text { detection } \\
\text { - overcome the conventional } \\
\text { threshold problem } \\
\text {-The false alarm rate is } \\
\text { comparatively low }\end{array}$ & \\
\hline 10 & \begin{tabular}{|c|} 
On-line Scene \\
Change Detection \\
of Multicast \\
Video
\end{tabular} & 2001 & $\begin{array}{c}\text { online } \\
\text { processing } \\
\text { (networking) }\end{array}$ & $\begin{array}{c}\text { Frame sampling } \\
\text { full decompression with } \\
\text { luminance } \\
\text { histogram scene change } \\
\text { detection algorithm } \\
\text { Partial decompression } \\
\text { algorithms }\end{array}$ & $\begin{array}{c}\text {-Ability to support real-time } \\
\text { video processing on the } \\
\text { network. } \\
- \\
\text { Capable of satisfying on-line } \\
\text { annotation needs }\end{array}$ & $\begin{array}{l}\text {-improving processing } \\
\text { speed and reducing } \\
\text { latency makes it improper } \\
\text { to compare to this } \\
\text { algorithms with other off- } \\
\text { line scene detection } \\
\text { technique } \\
\text {-complicated nature of } \\
\text { video processing }\end{array}$ \\
\hline
\end{tabular}


International Journal of Science and Research (IJSR)
ISSN (Online): 2319-7064

Index Copernicus Value (2013): 6.14 | Impact Factor (2014): 5.611

\begin{tabular}{|c|c|c|c|c|c|c|}
\hline & & & & & & \begin{tabular}{|c}
-plane to study of tolerant \\
of packet losses in the \\
network \\
- requirements of real- \\
time video multicasting \\
over the Internet \\
\end{tabular} \\
\hline 11 & $\begin{array}{c}\text { Scene change } \\
\text { detection by audio } \\
\text { and video clues }\end{array}$ & 2002 & $\begin{array}{l}\text { Multimedia } \\
\text { processing } \\
\text { (Informatio) }\end{array}$ & $\begin{array}{c}\text { Innovative } \\
\text { method for video shot } \\
\text { detection method }\end{array}$ & -Method performs very well & \\
\hline 12 & \begin{tabular}{|c|} 
Statistical \\
Sequential \\
Analysis for Real- \\
Time Video Scene \\
Change Detection \\
on \\
Compressed \\
Multimedia \\
Bitstream \\
\end{tabular} & 2003 & $\begin{array}{l}\text { multimedia } \\
\text { processing }\end{array}$ & $\begin{array}{l}\text { statistical sequential } \\
\text { analysis theory }\end{array}$ & $\begin{array}{l}\text {-Real-time approach } \\
\text {-Perform relatively well for } \\
\text { detecting }\end{array}$ & \begin{tabular}{|} 
For increased efficiency \\
original video data is \\
reduced using an optimal \\
transformation \\
,( low-dimensional \\
representation)
\end{tabular} \\
\hline 13 & \begin{tabular}{|c|} 
Efficient Scene \\
Change Detection \\
in MPEG \\
Compressed \\
Video Using \\
Composite Block \\
Averaged \\
Luminance Image \\
Sequence \\
\end{tabular} & 2005 & \begin{tabular}{|c|} 
Signal \\
Processing \\
(video indexing \\
\& segmentation)
\end{tabular} & 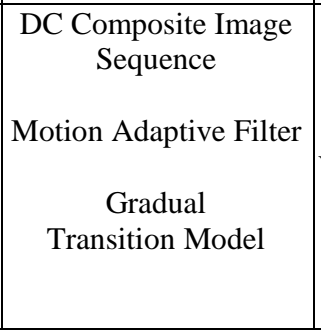 & \begin{tabular}{|c|}
-Lower complexity \\
-Better performance \\
Video indexing is widely used \\
on video database and video \\
available on the World Wide \\
Web (WWW).
\end{tabular} & $\begin{array}{l}\text { Using the DC composite } \\
\text { image alone is not precise } \\
\text { enough for scene change } \\
\text { detection }\end{array}$ \\
\hline 14 & $\begin{array}{c}\text { Fast Pixel-Based } \\
\text { Video Scene } \\
\text { Change Detection }\end{array}$ & 2005 & $\begin{array}{c}\text { Image } \\
\text { processing }\end{array}$ & \begin{tabular}{|} 
a two-phase reject-to- \\
refine strategy \\
is used. \\
rejecting a large portion \\
of easily detected non- \\
scene \\
change frames using \\
MAFD (mean absolute \\
frame differences) \\
further refining the \\
detection \\
process for the rest of \\
the frames.
\end{tabular} & \begin{tabular}{|}
-Method does not demand \\
time-consuming operations \\
such as motion estimation or \\
transform. \\
-Pixel-based methods have the \\
advantage on the ease of \\
computation and their \\
availability before actual \\
encoding \\
-Robustness \\
-Computational complexity is \\
low
\end{tabular} & $\begin{array}{l}\text { This technique is simple } \\
\text { but it performs poorly in } \\
\text { cases } \\
\text { of high motions of } \\
\text { objects, high motions of } \\
\text { cameras, or severe } \\
\text { scene luminance } \\
\text { variations (i.e., flicker) }\end{array}$ \\
\hline 15 & $\begin{array}{c}\text { PCA-based } \\
\text { approach for } \\
\text { video scene } \\
\text { change detection } \\
\text { on compressed } \\
\text { video }\end{array}$ & $\begin{array}{l}\text { AUG } \\
2006\end{array}$ & $\begin{array}{c}\text { Signal } \\
\text { Processing }\end{array}$ & $\begin{array}{c}\text { principal component } \\
\text { analysis }\end{array}$ & $\begin{array}{c}\text { superior performance } \\
\text { compared to the histogram } \\
\text { feature and the pixel feature. } \\
\text { PCA feature is then designed } \\
\text { to detect both abrupt and } \\
\text { gradual transitions. } \\
\text { An automatic, real-time } \\
\text { detection approach }\end{array}$ & $\begin{array}{c}\text { Tested on the TREC video } \\
\text { test repository to } \\
\text { validation for its } \\
\text { performance }\end{array}$ \\
\hline 16 & $\begin{array}{c}\text { Video scene } \\
\text { change detection } \\
\text { using neural } \\
\text { network: } \\
\text { Improved ART2 } \\
\end{array}$ & 2006 & neural network & $\begin{array}{l}\text { local minimum } \\
\text { detection algorithm }\end{array}$ & $\begin{array}{c}\text { reduce the small peaks such as } \\
\text { noises } \\
\text { constant scene were extracted. }\end{array}$ & $\begin{array}{c}\text { false detection when a big } \\
\text { object within the frame } \\
\text { was moving. }\end{array}$ \\
\hline 17 & \begin{tabular}{|} 
Adaptive group- \\
of-pictures and \\
scene change \\
detection methods \\
based on existing \\
H.264 advanced \\
video coding \\
information
\end{tabular} & $\begin{array}{l}\text { Sept } \\
2007\end{array}$ & $\begin{array}{c}\text { Image } \\
\text { Processing }\end{array}$ & $\begin{array}{c}\text { AGD( adaptive GOP } \\
\text { detection) and SCD } \\
\text { (scene change detection) } \\
\text { algorithms }\end{array}$ & \begin{tabular}{|} 
change detection rate of $98 \%$. \\
increase PSNR by at least 0.62 \\
dB \\
increase the
\end{tabular} & $\begin{array}{c}\text { existing VCV (video } \\
\text { content variations) } \\
\text { characteristics can be } \\
\text { further extended for } \\
\text { adaptive search window, } \\
\text { adaptive search methods }\end{array}$ \\
\hline 18 & $\begin{array}{l}\text { An Effective } \\
\text { Error } \\
\text { Concealment } \\
\text { Framework For }\end{array}$ & 2007 & $\begin{array}{l}\text { Image and } \\
\text { Graphics }\end{array}$ & $\begin{array}{l}\text { Weighted Forward } \\
\text { Motion Tracking } \\
\text { (WFMT) }\end{array}$ & \begin{tabular}{|c|} 
better robustness \\
efficiently improve the visual \\
quality and PSNR (1 to $3 \mathrm{~dB}$ \\
PSNR
\end{tabular} & $\begin{array}{l}\text { emphasize more on } \\
\text { exploring the scene } \\
\text { change algorithm for B } \\
\text { frames and improve }\end{array}$ \\
\hline
\end{tabular}




\section{International Journal of Science and Research (IJSR) \\ ISSN (Online): 2319-7064}

Index Copernicus Value (2013): 6.14 | Impact Factor (2014): 5.611

\begin{tabular}{|c|c|c|c|c|c|c|}
\hline & \begin{tabular}{|c|} 
H.264 Decoder \\
Based on \\
Video Scene \\
Change Detection \\
\end{tabular} & & & & substantial improvement) & $\begin{array}{l}\text { spatial or temporal EC } \\
\text { algorithms in the new } \\
\text { frame work }\end{array}$ \\
\hline 19 & \begin{tabular}{|} 
Fast Frame-Based \\
Scene Change \\
Detection in the \\
Compressed \\
Domain for \\
MPEG-4 Video
\end{tabular} & 2008 & $\begin{array}{c}\text { Mobile } \\
\text { Applications } \\
\text { Services and } \\
\text { Technologies }\end{array}$ & $\begin{array}{l}\text { a fast frame-based } \\
\text { algorithm }\end{array}$ & \begin{tabular}{|c|} 
easy computation \\
detection algorithm very fast \\
real time video processing \\
detects a high number of scene \\
changes and movements
\end{tabular} & $\begin{array}{c}\text { highly statistical nature of } \\
\text { the scene change detection } \\
\text { results design cannot be } \\
\text { absolutely correct }\end{array}$ \\
\hline 20 & $\begin{array}{l}\text { Video scene } \\
\text { detection using } \\
\text { graph-based } \\
\text { representations }\end{array}$ & $\begin{array}{l}\text { June } \\
2010\end{array}$ & $\begin{array}{l}\text { Signal } \\
\text { Processing- } \\
\text { Image } \\
\text { Communication }\end{array}$ & $\begin{array}{c}\text { graph-based video scene } \\
\text { boundary detection } \\
\text { method }\end{array}$ & $\begin{array}{l}\text { a fast, video scene detection } \\
\text { method } \\
\text { over all complexity is linear }\end{array}$ & $\begin{array}{l}\text { account for future } \\
\text { research directions }\end{array}$ \\
\hline 21 & \begin{tabular}{|c|} 
Disparity Map \\
Refinement for \\
Video Based \\
Scene Change \\
Detection Using a \\
Mobile \\
Stereo Camera \\
Platform \\
\end{tabular} & 2010 & $\begin{array}{c}\text { Pattern } \\
\text { Recognition }\end{array}$ & $\begin{array}{l}\text { a novel disparity map } \\
\text { refinement method }\end{array}$ & $\begin{array}{c}\text { detection of objects of } \\
\text { different sizes and textures in } \\
\text { real videos } \\
\text { minimizing false positives. }\end{array}$ & $\begin{array}{c}\text { future work will focus on } \\
\text { relaxing our assumption } \\
\text { on known temporal } \\
\text { alignment and improving } \\
\text { the spatial registration } \\
\text { module. }\end{array}$ \\
\hline 22 & $\begin{array}{c}\text { Hybrid Approach } \\
\text { for Video } \\
\text { Compression } \\
\text { Based on Scene } \\
\text { Change Detection }\end{array}$ & 2013 & $\begin{array}{c}\text { Signal } \\
\text { Processing }\end{array}$ & Hybrid Approach & $\begin{array}{l}\text { reduces the cost of } \\
\text { computational complexity }\end{array}$ & $\begin{array}{l}\text { Hybrid approach } \\
\text { compared with DS } \\
\text { algorithm } \\
\text { (Diamond search } \\
\text { algorithm )gives } \\
\text { approximately same } \\
\text { PSNR result } \\
\end{array}$ \\
\hline 23 & $\begin{array}{c}\text { Adaptive } \\
\text { Thresholding for } \\
\text { Scene Change } \\
\text { Detection }\end{array}$ & 2013 & $\begin{array}{l}\text { Signal } \\
\text { Processing - } \\
\text { Consumer } \\
\text { Electronics }\end{array}$ & $\begin{array}{c}\text { a novel adaptive } \\
\text { threshold decision } \\
\text { method }\end{array}$ & $\begin{array}{l}\text { stable in scene change } \\
\text { detection ability }\end{array}$ & $\begin{array}{c}\text { mean and variance of each } \\
\text { video sequences is } \\
\text { calculated for scene } \\
\text { change detection } \\
\text { measurement } \\
\end{array}$ \\
\hline 24 & \begin{tabular}{|c|} 
Performance \\
Evaluation of \\
Localized Person \\
Based Scene \\
Detection and \\
Retrieval in Video
\end{tabular} & 2013 & $\begin{array}{c}\text { Signal } \\
\text { Processing }\end{array}$ & $\begin{array}{c}\text { Block Based Chi-Square } \\
\text { histogram Comparison } \\
\text { Method }\end{array}$ & \begin{tabular}{|c|} 
The surveillance application \\
Human-computer interface \\
applications \\
Video content analysis \\
It is highly robust to camera, \\
objects motions \\
Recall and precision computed \\
values above $85 \%$ \\
Cartoon and animated movie \\
$91 \%$ F1 measure. \\
Movies dark screen $80 \%$ F1 \\
measure. \\
$100 \%$ results in advertisement \\
video.
\end{tabular} & $\begin{array}{l}\text { Improvement in its basic } \\
\text { inputs would result in } \\
\text { better performance of all } \\
\text { these techniques } \\
\text { Improvement was also } \\
\text { visible in critical (high } \\
\text { motion) boundary regions } \\
\end{array}$ \\
\hline 25 & $\begin{array}{c}\text { Video } \\
\text { watermarking } \\
\text { scheme based on } \\
\text { visual } \\
\text { cryptography } \\
\text { and scene change } \\
\text { detection }\end{array}$ & 2013 & Cryptography & \begin{tabular}{|c|} 
a novel video \\
watermarking scheme \\
based on visual \\
cryptography and scene \\
change detection in \\
discrete wavelet \\
transform domain \\
\end{tabular} & Robustness & $\begin{array}{l}\text { It is not possible to } \\
\text { recover the invisible } \\
\text { identification share } \\
\text { without the secret key in } \\
\text { the proposed scheme }\end{array}$ \\
\hline 26 & $\begin{array}{l}\text { Watermarking by } \\
\text { Adjusting the } \\
\text { Pixel Values and } \\
\text { Using Scene } \\
\text { Change Detection }\end{array}$ & 2013 & watermarking & a blind method & \begin{tabular}{|c|} 
robust against attacks such as \\
frame dropping, temporal \\
shifts and addition of noise. \\
The extraction process is blind \\
and the watermark can be \\
extracted without any \\
distortion from the
\end{tabular} & $\begin{array}{c}\text { Robustness of the scheme } \\
\text { can be further improved } \\
\text { by combining video with } \\
\text { audio watermarks } \\
\text { considerably robustness }\end{array}$ \\
\hline
\end{tabular}


International Journal of Science and Research (IJSR)

ISSN (Online): 2319-7064

Index Copernicus Value (2013): 6.14 | Impact Factor (2014): 5.611

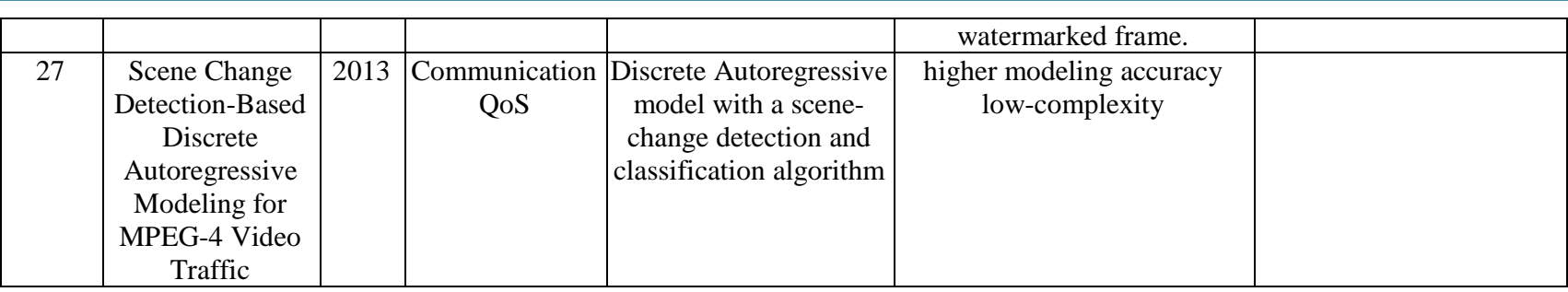

\subsection{Conclusion of Literature Survey}

The above survey of various researchers of different algorithms of Scene Change Detection shows that

PCA-based approach for video scene change detection on compressed video gives superior performance compared to the histogram feature and the pixel feature $\&$ is then designed to detect both abrupt and gradual transitions. But an automatic, real-time detection approach Tested on the TREC video test repository to validation for its performance. Neural Network based system reduces the small peaks such as noises constant scene were extracted but gives false detection when a big object within the frame was moving. Adaptive Thresholding method gives stability in scene change detection \& mean and variance of each video sequences is calculated for scene change detection measurement . Block Based Chi-Square histogram Comparison Method is highly robust to camera, objects motions. The Recall and precision computed values are above $85 \%$ \& gives $100 \%$ results but still some improvement in its basic inputs is required. Watermarking by Adjusting the Pixel Values and Using Scene Change Detection robust against attacks such as frame dropping, temporal shifts and addition of noise. and the watermark can be extracted without any distortion from the watermarked frame. But further improvement in the Robustness is required.

\section{Problem Identification}

\section{1) Complexity:}

- SCD Algorithmic complexity is concerned about how fast or slow particular SCD algorithm performs. A given algorithm will take different amounts of time on the same inputs depending on such factors as: processing speed .

- A ideal system is less complex in nature. Graph-based representations Scene Change Detection technique is complex rather than Adaptive Thresholding method and Histogram method.

\section{2) Sensitive in illumination changes-}

- Pixel-Based Video Scene Change Detection, this technique is performs poorly in cases of high motions of objects, high motions of cameras, or severe scene luminance variations (i.e., flicker) so that we Choose illuminationinsensitive color channels to avoid this problem.

\section{3) False Detection}

- Most of the algorithm suffer false detection with scenes involving fast camera or object motion. false alarm rate should be low.

- The measurement of the false detection based on precision and recall rate. Efficient scene change detector have the value of precision and recall are greater than $95 \%$.
4) PSNR :

- Peak signal-to-noise ratio is the important parameter of scene change detection algorithm, efficient SCD algorithm having the value of PSNR greater than 35 .

- All of the above techniques gives approximately same PSNR result or increase PSNR by at least same $\mathrm{dB}$.

\section{Methodology}

\subsection{Introduction}

The color histogram of an image can be computed by dividing a color space, e.g., RGB, into discrete image colors called bins and counting the number of pixels falling into each bin. The difference between two images Ii and Ij based on their color histograms $\mathrm{Hi}$ and $\mathrm{Hj}$ can be formulated as $\operatorname{diff}\left(I_{i}, I_{j}\right)=\sum_{k=1}^{n} \operatorname{abs}\left(H_{i k}-H_{j k}\right)$

The above relation denotes the difference in the number of pixels of two image that fall into same bin. In the RGB color space, the formula (1) can be written as

$\boldsymbol{d}_{R G B}\left(I_{i}, I_{j}\right)=\sum_{K}^{N}\left\{\operatorname{abs}\left[H_{i}^{r}(k)-H_{j i}^{r}(k)\right]+\operatorname{abs}\left[H_{i}^{r}(k)-\right.\right.$ $\left.\boldsymbol{H}_{j i}^{r}(\boldsymbol{k})\right]+\boldsymbol{a b s}\left[\boldsymbol{H}_{i}^{r}(\boldsymbol{k})-\boldsymbol{H}_{j i}^{r}(\boldsymbol{k})\right]$

Color histogram is used for detecting the changes in the successive video frames. Each video picture frame is converted into RGB image. For each RGB image $\boldsymbol{d}_{\boldsymbol{R} \boldsymbol{B} \boldsymbol{B}}\left(\boldsymbol{I}_{\boldsymbol{i}}, \boldsymbol{I}_{\boldsymbol{j}}\right)$ is calculated using equation (2).

The average of $\boldsymbol{d}_{\boldsymbol{R} \boldsymbol{G} \boldsymbol{B}}\left(\boldsymbol{I}_{\boldsymbol{i}}, \boldsymbol{I}_{\boldsymbol{j}}\right)$ is compared with a predefined threshold value. A value of $\boldsymbol{d}_{\boldsymbol{R} \boldsymbol{G} \boldsymbol{B}}\left(\boldsymbol{I}_{\boldsymbol{i}}, \boldsymbol{I}_{\boldsymbol{j}}\right)$ greater than threshold is selected. If average of $\boldsymbol{d}_{\boldsymbol{R} \boldsymbol{G} \boldsymbol{B}}\left(\boldsymbol{I}_{\boldsymbol{i}}, \boldsymbol{I}_{\boldsymbol{j}}\right)$ is greater than threshold, it means that a new scene has appeared by replacing the previous scene.

Proposed method is applied to frame difference as modifying log function and multiplying constant used to improve brightness of image in image processing.

$c=\frac{\max \left(d_{i f f l o g}\right)}{\max \left(\log \left(1+\operatorname{diff}^{2}\right)\right)}$

Where $d$ is the frame difference extracted from (2) and $c$ is the constant calculated from $d$. Square of frame difference is needed to show the difference value in dynamic range.

$\operatorname{diff}_{\log }=c \quad \log \left(1+d^{2}\right)$ 
International Journal of Science and Research (IJSR)

ISSN (Online): 2319-7064

Index Copernicus Value (2013): 6.14 | Impact Factor (2014): 5.611

4.2 Schematic Block Diagram of Proposed Methodology

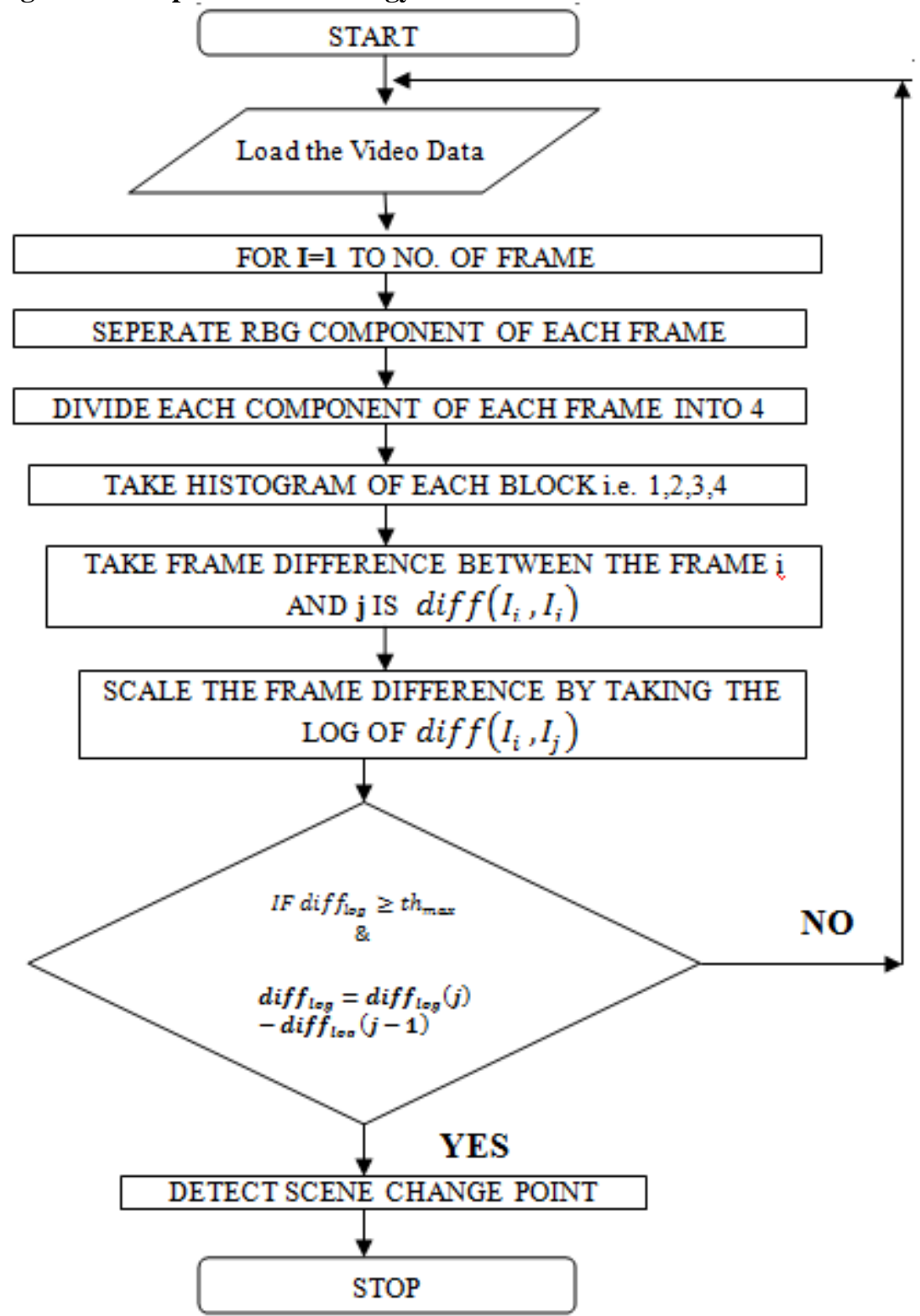

\subsection{Steps of the Proposed Method}

1) Load any video sequence.

2) Dive the video in number of frame.

3) Separate RGB color component of each and every frame.

4) Divide every component of each frame into four different blocks.

5) Take Histogram of each block i.e. 1, 2,3,4.

6) Take frame difference between the frame $\mathbf{i}$ and $\mathbf{j}$ is $\operatorname{diff}\left(I_{i}, I_{j}\right)$.

7) Scale the frame difference by taking the log of $\operatorname{diff}\left(I_{i}, I_{j}\right)$.

8) If this condition $\operatorname{diff}_{\text {log }}=\operatorname{diff}_{\log }(\boldsymbol{j})-\boldsymbol{d i f f}_{\log }(\boldsymbol{j}-\mathbf{1}) \& \operatorname{diff}_{\log } \geq$ $t h_{\max }$ is satisfy then go for forward processing to scene change detection point else it will go to load video data block.

\section{Expected Outcome}

In this project we have proposed new segmentation method based on color difference histogram. We have developed a new scene change detection method by scaling the histogram difference between the two frames. This method has presented an effective shot boundary detection algorithm for abrupt transition, which focuses on following difficult problems solution: i.e.

1) to provide the scaled frame difference that is dynamically compressed by log formula and it is more convenient to decide the threshold.

2) The shot boundary detection algorithm for abrupt transition gives stable SCD.

The proposed algorithm is promising, low false alarm, robustness where in gradual transition, camera fabrication, special events and so on can be incorporated. For a considerably good quality of scene change detection (SCD) algorithm are

- PSNR ranges should be $>40$. 


\section{International Journal of Science and Research (IJSR) \\ ISSN (Online): 2319-7064 \\ Index Copernicus Value (2013): 6.14 | Impact Factor (2014): 5.611}

- Recall \& precision rate $>95 \%$.

- Change detection rate $>98 \%$.

\section{Future Scope}

This project highlights and provides many important directions for future research. In this project we use in cryptography, face verification video indexing, Signal Processing Consumer Electronics. In future we apply our proposed algorithm for compress video sequences and Video Watermarking.

1) More work can be done on increasing the PSNR(Peak Signal to Noise Ratio) and precision \& Recall percentage.

2) We can also focus to reduce the processing time of the proposed algorithm.

\section{References}

[1] A. Nagasaka and Y. Tanaka, "Automatic Video Indexing and Full-Video Search for Object Appearances" Proceedings of the IFIP TC2/WG 2.6 Second Working Conference on Visual Database Systems II North-Holland Publishing Co. Amsterdam, The Netherlands Year-1992, pp113-127.

[2] H.J. Zhang, A. Kankanhalli, S.W. Smoliar, "Automatic Partitioning of Full-motion Video," in Multimedia Systems Volume-1, Issue-1, Year-1993 ACM-Springer, pp.10-28.

[3] K. Tse, J. Wei and S. Panchanathan "A Scene Change Detection Algorithm for MPEG Compressed Video Sequences" Canadian Conference on Electrical and Computer Engineering, Volume-2 Year-1995 IEEE ,pp. 827-830.

[4] Jianho Meng Yujen juan and Shih-Fu Chans "Scene change detection in a MPEG compressed video sequences," in Is\&T/Spie Symposium Proceedings Volume-2419, Year-1995,IEEE, pp.827-837.

[5] Jian FENG, Kwok-Tung LO and Hassan MEHRP'OUR "Scene Change Detection Algorithm for MPEG Video Sequence" in Image Processing on International Conference on Volume-1 Year-1996, IEEE, pp.821 824.

[6] Ralph M. Ford, Craig Robson, Daniel Temple, and Michael Gerlach "Metrics for Scene Change Detection in Digital Video Sequences"in IEEE International Conference on Multimedia Computing and Systems, Year-1997, IEEE, pp.610 - 611.

[7] Taehwan Shin, Jae-Gon Kim, Hankyu Lee and Jinwoong Kim "Hierarchical Scene Change Detection In An Mpeg-2 Compressed Video Sequence" in Circuits and Systems, Volume-4 , Year-1998, IEEE, pp.253 256.

[8] Haitao Jiang, Abdelsalam (Sumi) Helal, Ahmed K. Elmagarmid and Anupam Joshi "Scene change detection techniques for video database systems," in Multimedia System Springer-Verlag Volume-6, Issue-3, year-1998 , pp.86-195.

[9] W.A.C. Fernando, C.N. Canagarajah, D. R. Bull "Wipe Scene Change Detection in Video Sequences" in Image Processing, Volume-3, Year-1999, IEEE, pp.294-298.

[10]W. A. C. Fernando, C. N. Canagarajah and D. R. Bull "A Unified Approach To Scene Change Detection In
Uncompressed And Compressed Video "in Volume-46, issue-3, Year-2000, IEEE, pp.769-779.

[11]Wensheng Zhou1 and Asha Vellaikal "On-line Scene Change Detection of Multicast Video" in Journal of Visual Communication and Image Representation, Volume-3527, Year-2001, pp.1-15.

[12] Shu-Ching Chen, Mei-Ling Shyu, Wenhui Liao, Chengcui Zhang "Scene Change Detection By Audio And Video Clues" IEEE. Volume-2,Year-2002 , pp.365 - 368.

[13]Wing-San Chau, Oscar C. Au, Tak-Song Chong, TaiWai Chan, Chi-Shun Cheung "Efficient Scene Change Detection in MPEG Compressed Video Using Composite Block Averaged Luminance Image Sequence " in Fifth International Conference on Information, Communications and Signal Processing ,Year-2005 IEEE pp. 688 - 691.

[14] Xiaoquan Yi and Nam Ling "Fast Pixel-Based Video Scene Change Detection" in volume-4 Year-2005, IEEE , pp. 3443 - 3446.

[15] L. Gao, J. Jiang, J. Liang, S. Wang, S. Yang and Y. Qin "PCA-based approach for video scene change detection on compressed video" in Electronics Letters, Volume42, issue-24. Year-2006, pp.1389 - 1390 .

[16] Man-Hee Lee, Hun-Woo Yoo , Dong-Sik Jang "Video scene change detection using neural network: Improved ART2" in Elsevier Ltd., Volume-31, Issue 1, Year 2006, Pages 13-25.

[17]J.-R. Ding J.-F. Yang “Adaptive group-of-pictures and scene change detection methods based on existing H.264 advanced video coding information" in Image Processing, IET , Volume-2, Issue-2 Year-2008, pp. 85 94.

[18]ZHANG Ji, LIU Feng, SHAO Hui, WANG Gang "An Effective Error Concealment Framework For H.264 Decoder Based on Video Scene Change Detection" Fourth International Conference on Image and Graphics, Year-2007, IEEE, pp. 285 - 290.

[19] Jens Brandt Jens Trotzky Lars Wolf "Fast Frame-Based Scene Change Detection in the Compressed Domain for MPEG-4 Video " The Second International Conference on Next Generation Mobile Applications, Services, and Technologies, Year-2008, IEEE, pp.514 - 520

[20] Hakan Haberdar and Shishir K. Shah "Disparity Map Refinement for Video Based Scene Change Detection Using a Mobile Stereo Camera Platform" International Conference on Pattern Recognition, Year 2010 IEEE pp. 3890 - 3893.

[21]I. Spanou, A. Lazaris, and P. Koutsakis"Scene Change Detection-Based Discrete Autoregressive Modeling for MPEG-4 Video Traffic" in IEEE ICC 2013 Communication QoS, Reliability and Modeling Symposium, Year 2013 IEEE, pp. 2386 - 2390.

[22] Ankita P. Chauhan Rohit R.Parmar, Shankar K.Parmar Andshahida G.Chauhan "Hybrid Approach for Video Compression Based on Scene Change Detection" in Signal Processing, Computing and Control (ISPCC), Year-201, IEEE, pp.1-5

[23] Soongi Hong, Beobkeun Cho, and Yoonsik Choe "Adaptive Thresholding for Scene Change Detection" in Consumer Electronics IEEE Third International Conference on Consumer Electronics - Berlin (ICCEBerlin), Year-2013 , pp.75 - 78. 


\section{International Journal of Science and Research (IJSR) \\ ISSN (Online): 2319-7064}

Index Copernicus Value (2013): 6.14 | Impact Factor (2014): 5.611

[24] Neetirajsinh J. Chhasatia, Chintan U. Trivedi and Komal A. Shah "Performance Evaluation of Localized Person Based Scene Detection and Retrieval in Video " Image Information Processing (ICIIP), 2013 IEEE Second International Conference on Year- 2013 , pp. 78 - 83.

[25]S. Argyropoulos, P. List2, M.-N. Garcia, B. Feiten, M. Pettersson, and A. Raake "Scene Change Detection In Encrypted Video Bit Streams " in (ICIP) International Conference on Image Processing Year: 2013 , IEEE, pp. 2529 - 2533.

[26] Th. Rupachandra Singh, Kh. Manglem Singh, Sudipta Roy "Video watermarking scheme based on visual cryptography and scene change detection" in AEU International Journal of Electronics and Communications Volume 67, Issue-8, Year- 2013, pp. 645-651.

[27] Dolly Shukla, Chandra Shekhar Mithlesh and Manisha Sharma " Design, Implementation \& Analysis of Scene Change Detector using Block Processing Method" The Journal of Applied Sciences Research(TJASR), Volume- 2, No 2, 2015.pp-103-110.

[28] Dolly Shukla, Manisha Sharma and Chandra Shekhar Mithlesh, "A Survey On Different Video Scene Change Detection Techniques " International Journal Of Science And Research (IJSR), Volume-4, Issue-5, May2015,pp 214-219.

[29] Chandra shekhar Mithlesh, Dolley Shukla and Manisha Sharma "Video to Image Conversation Techniques-Key Frame Extraction,'International Journal of Emerging Science and Engineering, Volume-4, Issue- 3, March 25,2016,pp.30-34.

[30] Chandra shekhar Mithlesh and Dolley Shukla , "A Case Study of Key frame Extraction Techniques," International Journal of Advanced Research in Electrical, Electronics and Instrumentation Engineering, Vol. 5, Issue 3, March 2016.

\section{Author Prfile}

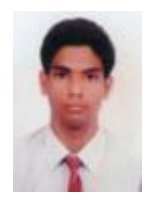

Chandra Shekhar Mithlesh is currently working as Asst. Professor in Dept. of Electronics and Telecommunication Engineering in Shri Rawatpura Group of Institution, New Raipur, Chhattisgarh. He is M.E. Scholar in Communication System Engineering in Shri Shankracharya Group of Institution, Bhilai, Chhattisgarh. He received the B.E degrees in Electronics and Telecommunication Engineering from Govt. Engineering College Jagdalpur, Chhattisgarh in 2009. He is having more than 3 years of experience in academics. He has published several papers at various national/International Journals/Conferences. He is a member of Indian Science Congress Association, ACM, IAENG and CSTA. His research interests are Image \& Video processing, Wireless communications, Antenna, Electromagnetic field, Embedded system. 